\title{
Rare mutations in the accessory proteins ORF6, ORF7b and ORF10 of the SARS-CoV2 genomes
}

\author{
Sk. Sarif Hassan ${ }^{\mathrm{a}, *}$, Pabitra Pal Choudhury ${ }^{\mathrm{b}}$, Bidyut Roy ${ }^{\mathrm{c}}$ \\ ${ }^{a}$ Department of Mathematics, Pingla Thana Mahavidyalaya, Maligram 721140, India \\ ${ }^{b}$ Applied Statistics Unit, Indian Statistical Institute, Kolkata 700108, West Bengal, India \\ ${ }^{c}$ Human Genetics Unit, Indian Statistical Institute, Kolkata 700108, West Bengal, India
}

\begin{abstract}
In the NCBI database, as on May 29, 2020, total number of available complete genome sequences of SARSCoV2 across the world is 3080. Every accessory proteins viz. ORF6, ORF7b and ORF10 possess a single missense mutation in less than of $1.5 \%$ of the 3080 genomes. Different non-synonymous mutations may affect the functions of the accessory proteins.
\end{abstract}

Keywords: ORF6; ORF7b; ORF10; Rare mutations;

\section{Introduction}

The accessory proteins of SARS-CoV2 help the virus to infect its human host, replicate and eventually spread from person to person. There are six accessory proteins ORF3a, ORF6, ORF7a, ORF7b, ORF8 and ORF10 in the SARS-CoV2 genomes, which play important roles in the viral life cycle and may contribute to the pathogenesis and virulence [1. These accessory proteins are unique to SARS-CoV2, as they have least homology in amino acid sequence with accessory proteins of other coronaviruses. These proteins contribute to virus virulence though it does not directly affect the virus release, stability and pathogenesis [1]. Therefore, close observations and understanding of these proteins would explain the differences in pathogenicity between SARS-CoV2 and other known coronaviruses. Within a very quick time span, the virus has been evolving for accounting various missense mutations in various proteins. It has been already observed by us that except one in ORF7b, there are no missense mutations over the proteins ORF6, ORF7b (except in one) and ORF10 across the SARS-C0V2 genomes of the 128 Indian patients [2, 3. So it is intended to discover whether any missense mutations are found in those proteins across the SARS-CoV2 genomes worldwide, other than India. Note that among 3080 SARS-CoV2 genomes, only $1.5 \%$ of the genomes contains the mutations in these three accessory proteins and hence we call it is as 'rare missense mutations'.

\subsection{Functions of the accessory proteins ORF6, ORFrb and ORF10}

Briefly we present the known functions of the three accessory proteins ORF6, ORF7b and ORF10 as follows.

\footnotetext{
* Corresponding author

Email addresses: sarimif@gmail.com (Sk. Sarif Hassan), pabitrapalchoudhury@gmail.com (Pabitra Pal Choudhury), broy@isical.ac.in (Bidyut Roy)
} 
- ORF6: The ORF6 gene encodes a protein with a hydrophobic N-terminal and that has been suggested to have a N-endo-C-endo conformation [4]. The ORF6 protein makes intracellular membrane rearrangements resulting in a vesicular population in the infected cell which could possibly serve some role in increasing replication of the virus [5]. It has been previously reported that ORF6 down-regulated the mRNA level of the co-transfected myc-nsp8 gene [6]. It is also reported that amino acids 53-56 in ORF6 of SARS-CoV constitute a putative diacidic motif (DDEE) which influences the suppression of the expression of co-transfected myc-nsp8 [4].

- ORF7b: The sequence of ORF7b is very much unique since it does not have any sequence homology with other viral proteins [7]. The accessory protein ORF7b has been reported to have a structural component in the SARS-CoV virions [8]. The transmembrane domain of ORF7b is essential to retain the protein in the Golgi compartment $[$. The ORF7b of SARS-CoV2 is $81 \%$ identical to that of the SARS-CoV1. It is reported that absence of ORF7b does not affect virus replication [8]. Alanine scanning experiments showed that the amino acids in 13-15 and 19-22 are critical for the retention of ORF7b in the Golgi complex [9].

- ORF10: ORF10 protein is the unique accessory protein in SARS-CoV2 as it does not present in the SARS-CoV1 [10] and hence it is sometimes called "mysterious protein". The signature appearance of ORF10 in SARS-CoV2 makes the novel coronavirus easily detectable than PCR based methods [11. Function of this protein is still undiscovered. Therefore any missense mutation in ORF10 is significantly worth to determine and require further investigations in order to understand the functions of ORF10.

\section{Methods}

All the protein sequences of 3080 SARS-CoV2 genomes are fetched from the NCBI virus database. Then for each of the three accessory proteins, amino acid sequences are exported in fasta format using file operations through Matlab. For each accessory protein, these sequences (fasta formatted) are blasted using ClustalOmega and found the mismatched and from there mutations and their associated positions are accounted [12.

\section{Results}

It is noted that among these virus genomes from 3080 patients, there are 2126 genomes from USA, 306 from Asia, 281 genomes from Europe, 365 genomes from Oceania and 1 genomes from Africa. Here (Table 1), we present the missense mutations of the three accessory proteins ORF6, ORF7b and ORF10 over the available 3080 SARS-CoV2 genomes. Note that the mutation of an amino acid $A_{1}$ to an amino acid $A_{2}$ is denoted by $A_{1}(l) A_{2}$ where $l$ denotes location in the reference amino acid sequence. In the Table 1 , also the changes of the R-group of each amino acid according to the mutations are presented. The Table- 1 depicts the following: 


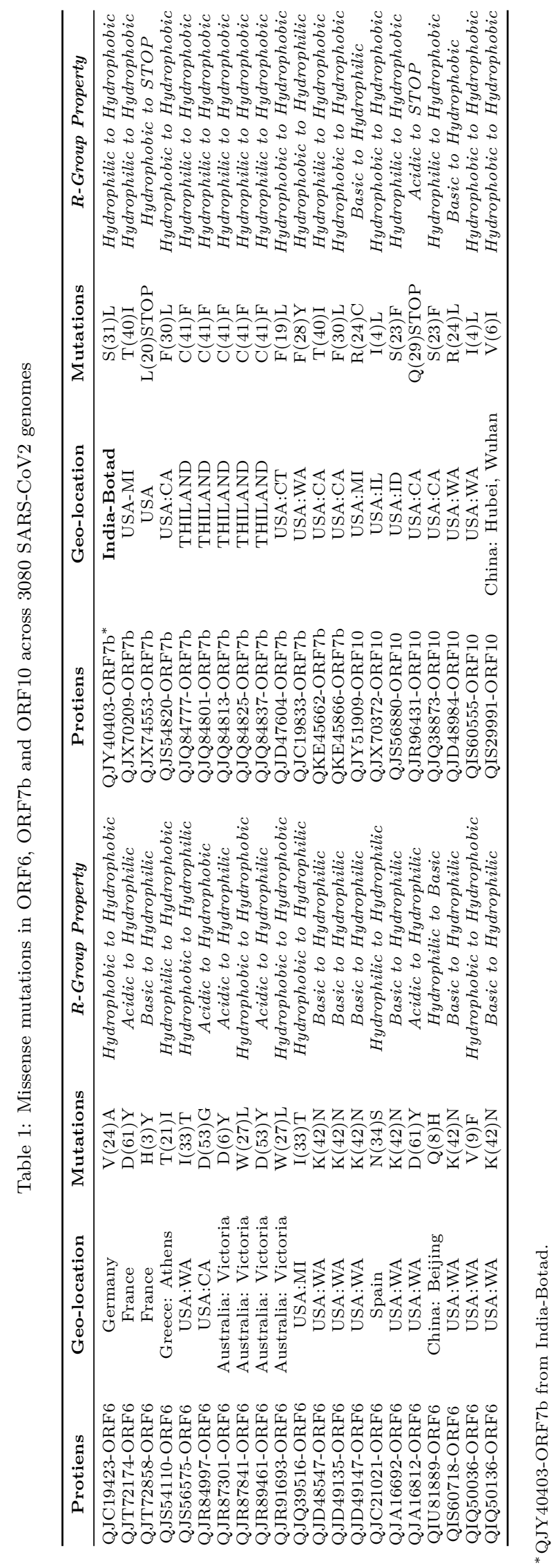


- In less than $1.5 \%$ of the SARS-CoV2 genomes, the accessory proteins ORF6 (1.49\%), ORF7b (0.422\%) and ORF10 (0.259\%) possess the missense mutations as adumbrated in the Table 1.

- The ORF7b protein (QJX74553) got truncated due to mutation L(20)STOP to a stop codon. Also the protein ORF10 (QJR96431) is truncated due to the mutation Q(29)STOP.

- There are several changes of the R-group property of amino acids viz. hydrophobic to hydrophobic, acidic to hydrophilic, basic to hydrophilic, hydrophilic to hydrophobic, acidic to hydrophobic, hydrophobic to hydrophilic, hydrophilic to basic and basic to hydrophobic.

- The putative diacidic motif (DDEE) in SARS-CoV of the ORF6 protein in the 53-56 position has been mutated to DEEQ in ORF6 of most of the SARS-CoV2 genomes. This motif DEEQ of ORF6 has been mutated $(\mathrm{D}(53) \mathrm{Y})$ to YEEQ in QJR89461-ORF6 (Australia: Victoria). Strikingly, the motif DEEQ mutated $(\mathrm{D}(53) \mathrm{G})$ to GEEQ in the ORF6 protein across the 25 SARS-CoV2 genomes from USA:CA as shown in Table 2. These missense mutations would certainly affect the suppression of the expression of co-transfected myc-nsp8.

Table 2: 25 SARS-CoV2 genomes from USA:CA having D(53)G mutation in ORF6

\begin{tabular}{lcc}
\hline \multicolumn{3}{c}{ USA $\boldsymbol{C A}$} \\
\hline \multicolumn{2}{c}{ Mutation: D(53)G } & Acidic to Hydrophobic \\
QKE49188-ORF6 & QKE49296-ORF6 & QKE49404-ORF6 \\
QKE49200-ORF6 & QKE49320-ORF6 & QKE49416-ORF6 \\
QKE49212-ORF6 & QKE49332-ORF6 & QKE49428-ORF6 \\
QKE49224-ORF6 & QKE49344-ORF6 & QKE49440-ORF6 \\
QKE49236-ORF6 & QKE49356-ORF6 & QKE49452-ORF6 \\
QKE49248-ORF6 & QKE49368-ORF6 & QKE49464-ORF6 \\
QKE49260-ORF6 & QKE49380-ORF6 & QKE49476-ORF6 \\
QKE49272-ORF6 & QKE49392-ORF6 & QKE49488-ORF6 \\
& & QKE49500-ORF6 \\
\hline
\end{tabular}

- The transmembrane domain of ORF7b in SARS-CoV2 is fully conserved with respect to that of SARSCOV. In the protein QJD47604-ORF7b (USA:CT) possesses a mutation F(19)L which might affect the retention of ORF7b in the Golgi complex. There is non-sense mutation L(20)STOP in the QJX74553ORF7b (USA), which makes the protein non-functional.

- The nonsense mutation in QJR96431-ORF10 (USA:CA) makes the protein non-functional. In the USA, three distinct missense mutations $\mathrm{R}(24) \mathrm{C}, \mathrm{I}(4) \mathrm{L}$ and $\mathrm{S}(23) \mathrm{F}$ are found in each pair of genomes viz. $\{Q J Y 51909-O R F 10, Q J D 48984-O R F 10\}$, $\{Q J X 70372-O R F 10, Q I S 60555-O R F 10\}$ and $\{Q J S 56880$ - ORF10, QJQ38873 - ORF10\} respectively. Also there is another mutation V(6)I (hydrophobic to hydrophobic) in QIS29991-ORF10 (China: Hubei, Wuhan) which might not affect the functions of ORF10 because of synonymous change of R-group property. 


\section{Concluding remarks}

The novel RNA virus have been evolving through several mutations over the associated proteins within a very short time from its discovery since December, 2019 though ORF6, ORF7b and ORF10 were 100\% conserved. It has now been observed that there are some mutations happening in the these three proteins too. Most of these rare mutations are changing the amino acids such as hydrophilic to hydrophobic, acidic or basic to hydrophobic and vice versa etc. So these mutations might have significant effect on the corresponding protein function. This study opens a question whether it carries some messages about the virus rapid replications and virulence and so on. Whether these mutations raise different symptoms in the affected patients, is certainly of worth investigation from the pathogenesis perspective. These rare mutations may suggest to develop antiviral drugs or formulate new vaccines from a new starting point. It will also be interesting to explore whether these rare mutations were evolved in China or other affected countries. Although the clinical importance of these rare mutations are yet to be discovered, this study is certainly would serve as a groundwork.

\section{Author Contributions}

SH conceived the problem. SH determined the mutations. SH, PPC, BR analysed the data and result. SH wrote the initial draft which was checked and edited by all other authors to generate the final version.

\section{Conflict of Interests}

The authors do not have any conflicts of interest to declare.

\section{References}

[1] K. Xu, B.-J. Zheng, R. Zeng, W. Lu, Y.-P. Lin, L. Xue, L. Li, L.-L. Yang, C. Xu, J. Dai, et al., Severe acute respiratory syndrome coronavirus accessory protein 9b is a virion-associated protein, Virology 388 (2) (2009) 279-285.

[2] S. S. Hassan, P. P. Choudhury, B. Roy, S. S. Jana, Missense mutations in sars-cov2 genomes from indian patients (May 2020). doi:10.31219/osf.io/2wm8h

URL osf .io/2wm8h

[3] S. S. Hassan, P. P. Choudhury, P. Basu, S. S. Jana, Molecular conservation and differential mutation on orf3a gene in indian sars-cov2 genomes, BioRxiv (2020).

[4] V. Gunalan, A. Mirazimi, Y.-J. Tan, A putative diacidic motif in the sars-cov orf6 protein influences its subcellular localization and suppression of expression of co-transfected expression constructs, BMC research notes 4 (1) (2011) 446. 
[5] M. Frieman, B. Yount, M. Heise, S. A. Kopecky-Bromberg, P. Palese, R. S. Baric, Severe acute respiratory syndrome coronavirus orf6 antagonizes stat1 function by sequestering nuclear import factors on the rough endoplasmic reticulum/golgi membrane, Journal of virology 81 (18) (2007) 9812-9824.

[6] H. Zhou, D. Ferraro, J. Zhao, S. Hussain, J. Shao, J. Trujillo, J. Netland, T. Gallagher, S. Perlman, The n-terminal region of severe acute respiratory syndrome coronavirus protein 6 induces membrane rearrangement and enhances virus replication, Journal of virology 84 (7) (2010) 3542-3551.

[7] S. R. Schaecher, J. M. Mackenzie, A. Pekosz, The orf7b protein of severe acute respiratory syndrome coronavirus (sars-cov) is expressed in virus-infected cells and incorporated into sars-cov particles, Journal of virology 81 (2) (2007) 718-731.

[8] A. Pekosz, S. R. Schaecher, M. S. Diamond, D. H. Fremont, A. C. Sims, R. S. Baric, Structure, expression, and intracellular localization of the sars-cov accessory proteins $7 \mathrm{a}$ and $7 \mathrm{~b}$, in: The Nidoviruses, Springer, 2006, pp. 115-120.

[9] S. R. Schaecher, M. S. Diamond, A. Pekosz, The transmembrane domain of the severe acute respiratory syndrome coronavirus orf7b protein is necessary and sufficient for its retention in the golgi complex, Journal of virology 82 (19) (2008) 9477-9491.

[10] G. Taiaroa, D. Rawlinson, L. Featherstone, M. Pitt, L. Caly, J. Druce, D. Purcell, L. Harty, T. Tran, J. Roberts, et al., Direct rna sequencing and early evolution of sars-cov-2, bioRxiv (2020).

[11] T. Koyama, D. Platt, L. Parida, Variant analysis of covid-19 genomes, Bulletin of the World Health Organization (2020).

[12] F. Madeira, Y. M. Park, J. Lee, N. Buso, T. Gur, N. Madhusoodanan, P. Basutkar, A. R. Tivey, S. C. Potter, R. D. Finn, et al., The embl-ebi search and sequence analysis tools apis in 2019, Nucleic acids research 47 (W1) (2019) W636-W641. 